\title{
Tibetan Medicines for the Treatment of Diabetic Nephropathy
}

\author{
Lili Pu $\mathbb{D}^{1},{ }^{1}$ Chunhong Yang, ${ }^{1}$ Liqiong Yu, ${ }^{1}$ Shiling Li, ${ }^{1}$ Yaqin Liu, ${ }^{1}$ Xinan Liu, \\ and Xianrong Lai $\mathbb{D}^{1,2}$ \\ ${ }^{1}$ School of Pharmacy, Chengdu University of Traditional Chinese Medicine, Chengdu 611137, China \\ ${ }^{2}$ School of Ethnic Medicine, Chengdu University of Traditional Chinese Medicine, Chengdu 611137, China \\ Correspondence should be addressed to Xianrong Lai; 105114018@qq.com
}

Received 10 August 2021; Revised 6 September 2021; Accepted 9 September 2021; Published 6 October 2021

Academic Editor: Harry Lee

Copyright ( 2021 Lili Pu et al. This is an open access article distributed under the Creative Commons Attribution License, which permits unrestricted use, distribution, and reproduction in any medium, provided the original work is properly cited.

As an important part of the traditional Chinese medicine system, Tibetan medicine has its unique treatment methods for diabetes mellitus and its complications. Diabetic nephropathy (DN) is one of the most serious diabetic microvascular diseases. Tibetan medicine believes that the occurrence of DN is closely related to renal function changes, and it can be effectively prevented and treated by improving renal lesions. In this paper, we consult ancient books of Tibetan medicine and summarize the medicines that treat kidney disease in the Tibetan medicine system. The Chinese name, English name, and Latin name of these drugs were searched as keywords in the online database. Thirty-four drugs were found for the treatment of DN. The most commonly used were Amomum kravanh, Terminalia chebula, and Tribulus terrestris, and we introduced the traditional uses and modern pharmacological activities of these drugs. The results indicate that Tibetan medicines for kidney disease could be used as potential candidate drugs for DN; they would expand the range of medications for DN and provide a new idea for the treatment of DN.

\section{Introduction}

Diabetic nephropathy (DN) is a series of microvascular complications caused by changes in renal structure and function due to chronic diabetic microangiopathy. It is mainly characterized by continuous albuminuria and progressive reductions in renal function. It is one of the most serious chronic complications of diabetes mellitus and one of the most common causes of end-stage renal disease (ESRD) [1]. If it is not treated promptly, it will endanger the life and health of patients. According to relevant epidemiological statistics, diabetic patients are expected to reach 578 million in the world by 2030; meanwhile, DN patients will break through 100 million [2]. The incidence of DN in diabetic patients in China is about $47.66 \%$ [3]. Its pathological features include early glomerular hyperfiltration, changes in capillary permeability, increase in mesangial membrane cells and matrix, glomerular basement membrane thickening, deposition of extracellular matrix, subsequent glomerular sclerosis and fibrosis, and ultimately leading to the deterioration of renal function [4], which is the main cause of death in diabetic patients. The pathogenesis of DN is complex. Modern medicine mainly prevents and treats DN by controlling blood glucose and blood pressure, regulating blood lipids, lowering urinary albumin, and following other principles. But diabetes mellitus and its complications cannot be treated well by these simple means only $[5,6]$. So it is necessary to combine modern medical treatments with traditional medical methods to obtain better therapeutic results.

Tibetan medicine is an important part of the treasure house of Chinese medicine, which has a long history, complete theory, and rich content. It has made great contributions to the reproduction, survival, and development of Tibetan people and has a unique curative effect on the treatment of diabetes mellitus and its complications. Based on the knowledge of DN from the Tibetan medicine theory, we have found that the occurrence of DN is closely related to kidney lesions. And modern studies have also found that DN can be effectively prevented and treated by protecting kidneys and improving kidney function [7, 8]. Tibetan medicine has a unique therapeutic approach to diabetes 
mellitus and its complications and has been gradually applied in the treatment of DN. However, the current records on Tibetan medicines for the treatment of DN are not comprehensive enough and lack systematic summary and generalization. Therefore, supported by Tibetan medicine theory, we attempt to find Tibetan medicines for DN from the Tibetan medicines for treating kidney diseases, in order to expand the drug use range of DN and provide new ideas for the treatment of DN.

\section{The Knowledge of Tibetan Medicines on DN}

Tibetan medicine believes that there are three major factors: rLung, Bad-kan, and mKhris-pa in the physiological activities of the human body. The three factors control the movement changes of the seven material bases (seven essences) in diet essence, blood, meat, fat, bone, bone marrow, semen, and the motion of three excretions (three filth) in stool, urine, and sweat. Under certain conditions, the three basic factors are relatively coordinated, maintaining the balance with the seven essences and the three obscenities and keeping the normal physiological activities of the body. Once one of the three factors changes, this balance will be disrupted, causing various pathological changes that can lead to disease.

DN belongs to the category of "jingnisaku disease" (Chinese direct translation: frequent urination) in Tibetan medicine, and Tibetan medicine believes that "jingnisaku disease" is a consumptive disease, which promotes the consumption and reduction of mKhris-pa (cold), thus losing its counterbalancing effect on Bad-kan (hot) and increasing the effect of Bad-kan. The decrease in mKhris-pa and the increase in Bad-kan lead to the dysfunction of rLung, which makes the originally reduced mKhris-pa in the body or tissues decrease more and more, while the originally increased Bad-kan increases more and more, and the imbalance of the three factors is getting worse, then driving the further deterioration of DN. The "jingnisaku disease" is caused by the imbalance of rLung, Bad-kan, and mKhris-pa due to external factors such as improper diet and living. The ancient book of Tibetan medicine Rgyud bzhi records: "due to eating salty, sweet, cold, or heavy diet, living in a humid place for a long time, growing Bad-kan size, cannot be sublimated to the essence to be absorbed and leak into the bladder, producing body fluids and causing disease." In the Blue Glaze, it is said that "long-term living in damp places and other reasons weaken the function of the kidneys, unable to differentiate the dregs and essences, directly fall into the bladder, and cause frequent urination." This is similar to modern medicine, which believes DN is dominated by persistent proteinuria and accompanied by varying degrees of frequent urination, acute pain, and urinary discomfort. Tibetan medicine believes that "jingnisaku disease" can be caused by factors such as weakened kidney function. The prescriptions for the treatment of "jingnisaku disease," such as Shibawei Hezi diuretic pill and Shiliu Rilun pill, have the effect of benefiting the kidneys. And Tribulus terrestris, a Tibetan medicine for the treatment of kidney disease, has also been used in the Tibetan formula Siwei Jianghuang decoction powder for the treatment of "jingnisaku disease." In conclusion, the occurrence of "jingnisaku disease" is closely related to kidney pathology.

\section{Materials and Methods [9]}

We have manually searched eleven Tibetan medicine monographs and drug standards, such as Dictionary of Chinese Ethnic Medicine, Jing Zhu Materia Medica, Chinese Tibetan Materia Medica, Rgyud bzhi, and Blue Glaze. And we have looked up the information on Tibetan medicines for the treatment of kidney diseases and used their dialect, English, or Latin names as keywords to search in Chinese online databases (such as CNKI, VIP, and Wanfang) and English online databases (such as Sci-Hub, ScienceDirect, GeenMedical, etc.) to obtain their modern pharmacological studies on DN. In addition, we consulted the Tibetan prescriptions for the treatment of renal diseases in Treasure House of Tibetan Medicine Prescriptions and Interpretation of Commonly Used Tibetan Medicines and summarized the commonly used Tibetan medicines that are used more frequently and can treat DN from these formulas.

\section{Results}

In this paper, we reviewed 362 Tibetan medicines for the treatment of kidney diseases (nephritis, kidney deficiency, kidney cold, kidney heat, kidney edema, etc.) in the traditional Tibetan medicine system. Among these 362 Tibetan medicines, 60 have been used to treat kidney diseases in modern studies, of which 34 have been used for the treatment of DN. The 34 Tibetan medicines for DN are all botanicals, distributed in 23 different families, and the most common families are Leguminosae (23\%), Umbelliferae (6\%), Zingiberaceae (6\%), Rubiaceae (6\%), and Solanaceae (6\%) (Figure 1). The scientific names, Chinese names, Tibetan names, families, medicinal parts, and modern pharmacological effects of the Tibetan medicines for DN are shown in Table 1. In addition, we have found that 16 Tibetan medicines for the treatment of kidney diseases have antidiabetic activity. Therefore, it is necessary to further study the effects of these Tibetan medicines on DN, with a view to using them in the treatment of $\mathrm{DN}$.

4.1. Tibetan Medicines for Treating DN. It can be seen from Table 1 that the above Tibetan medicines can protect the kidney by improving the expression of related factors (TGF, HIF-1 $\alpha$, VEGF, SOD, etc.) in renal tissue, inhibiting the damage of renal epithelial cells, alleviating renal fibrosis, scavenging free radicals, and inhibiting lipid peroxidation and other ways, and play a role in the treatment of DN.

4.2. High-Frequency Tibetan Medicines. In order to understand the frequency of Tibetan medicines use for the treatment of DN, we also inquired about Tibetan medicine prescription books such as Treasure House of Tibetan Medicine Prescriptions and Interpretation of Commonly Used Tibetan Medicines and collected 123 Tibetan medicine 


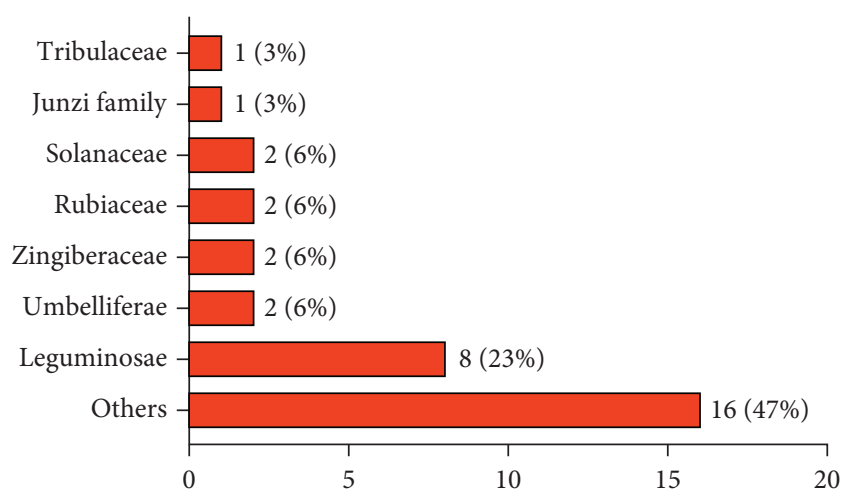

Figure 1: The most common families of Tibetan medicines in the treatment of $\mathrm{DN}$.

prescriptions for kidney disease. The Traditional Chinese Medicine Inheritance Support System (version 2.5) [74] is used to get the frequency of Tibetan medicines used in prescriptions. Through data mining, there are 7 drugs that are used more than 20 times and can treat DN (Figure 2); they are Amomum kravanh with the used frequency of 54, Terminalia chebula with 42, Malva verticillata with 40, Rubia cordifolia with 29, T. terrestris with 27, Piper longum with 25, Punica granatum with 22.

The following is a detailed introduction to the name base sources, traditional effects, and modern pharmacological effects of the three most widely used Tibetan medicines (A. kravanh, T. chebula, and T. terrestris).

4.2.1. Amomum kravanh. This is the dried ripe fruit of the Zingiberaceae plant A. kravanh Pierre ex Gagnep (Tibetan name: $\mathbb{N}_{\circlearrowleft} \nabla^{\top} \mathbb{N}_{\text {म }}$ ता (transliterated as Jia-na-su-men) and English name: Amomum cardamomum). It is originated in Cambodia and Thailand and is now introduced and cultivated in Yunnan and Guangdong in China, mostly in the wet area of the trench. It is applied for the treatment of diseases such as waist and leg soreness caused by kidney cold in Tibetan clinics (Chinese Materia Medica-Tibetan Medicine Roll). Blue Glaze records that A. cardamomum treats cold nephropathy and various diseases caused by rLung. In Rgyud $b z h i$, A. cardamomum can cure kidney disease and all cold diseases.

A. cardamomum is widely used in the treatment of kidney diseases, and it is also recorded in Tibetan medicine. The main chemical component of A. cardamomum is a volatile oil, which has various pharmacological activities such as antibacterial, antioxidation, and hypoglycemic activity. A. cardamomum is the main medicine of Tibetan medicine compound preparations (such as Shiwei Cardamom Pill and Shibawei Hezi diuretic pill) for the treatment of kidney diseases. The modern use of cardamom has also shown some efficacy in treating many kidney diseases such as chronic renal failure, chronic pyelonephritis, and DN. Chen et al. [75] found that cardamomum volatile oil can reduce the urine protein content of acute kidney injury rats; reduce renal body index; improve renal tubular pathology; reduce MDA, NO content, and NOS activity in renal tissue; and increase SOD and GSH-Px activity in the kidney tissues so that cardamomum can improve the acute kidney injury of rats caused by gentamicin to a certain extent. Chen et al. [76] showed that $A$. cardamomum can reduce kidney damage in rats with adriamycin nephropathy, and its mechanisms may be related to the expression of TGF- $\beta 1$ and PAI- 1 in kidney tissue. They also found that $A$. cardamomum volatile oil can upregulate MMP-2 TGF- $\beta 1$ and IGF-2 protein expression, significantly reduce the blood glucose of streptozotocininduced DN model rats, and improve the kidney pathological changes in DN rats. In conclusion, A. cardamomum has a certain therapeutic effect on DN, but there are relatively few studies on its mechanisms. Therefore, modern analytical methods should be used to conduct a more extensive and in-depth experimental study on the effects and mechanisms of $A$. cardamomum in the treatment of $\mathrm{DN}$, making it a common and effective drug for the treatment of DN.

4.2.2. Terminalia chebula. This is the fruit of the Junzi family plant T. chebula Retz. (Tibetan name: ${ }^{\top}{ }^{\top}{ }^{\top}$ (transliterated as A-ru-re)). Born in the sparse forest at an altitude of $800-1000 \mathrm{~m}$, mainly distributed in western and southwestern Yunnan, also cultivated in Guangdong and Guangxi. Rgyud $b z h i$ records that $T$. chebula has six flavors, eight properties, and seventeen functions. Known as the "King of Tibetan Medicine" in China, it can be used in the treatment of kidney disease, diabetes, and other diseases in the Tibetan medicine system.

The effective components of T. chebula are complex, of which phenolic acids are the main components; it has many pharmacological activities such as antioxidation, antidiabetic, antipathogenic microorganism, anti-inflammatory, analgesic, and so on [77]. T. chebula has a strong antioxidant capacity, which is the basis of its efficacy in protecting kidney function and preventing diabetes, and studies have found that polyphenols are the material basis for the antioxidant effect of T. chebula [78]. Tayal et al. [79] found that the aqueous extract of $T$. chebula had a protective effect against oxalate-induced injury to NRK-52E and MDCK renal epithelial cells; it can enhance cell viability, reduce LDH release, and inhibit the nucleation and growth of calcium oxalate crystals, thus playing a role in protecting the kidneys. Rao and Nammi [80] found that T. chebula extract can reduce blood glucose in streptozotocin-induced diabetic rats, has strong antidiabetic and renal protection, and can be used for the treatment of DN. Kim et al. [81] showed that T. chebula extract can reduce blood glucose, blood lipids and serum MAD levels in diabetic rats caused by streptozotocin, improve the kidney pathological tissue morphology, and reduce the formation of AGEs in diabetic rats. Li et al. [11] found that $T$. chebula acid can affect the phosphorylation of VEGF-2, which cannot normally promote angiogenesis after VEGF binding to the receptor, thereby inhibiting the progression of DN. Therefore, $T$. chebula cannot only protect the kidneys and prevent and treat diabetes but also be used in the treatment of DN. It is the first choice for Tibetan medicine to treat various diseases. 
TABLE 1: Tibetan medicines for DN in modern research (the order of Tibetan medicine names is from high to low according to the frequency of use).

\begin{tabular}{lcc}
\hline No. Latin name & $\begin{array}{c}\text { Chinese } \\
\text { name }\end{array}$ & $\begin{array}{c}\text { Tibetan } \\
\text { name }\end{array}$
\end{tabular} Family Medication part Modern study on the treatment of DN

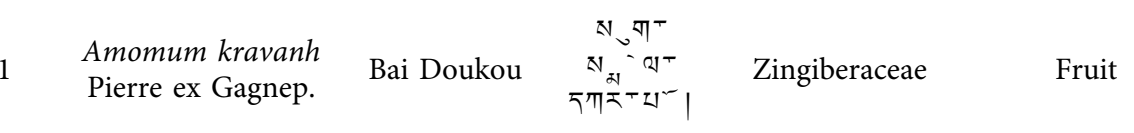

White cardamom volatile oil upregulates the expression of MMP-2, TGF- $\beta 1$, and IGF-2, thereby improving the pathological changes of DN caused by streptozotocin (STZ) and protecting the kidneys of diabetic rats [10].

Chebulinic acid can affect the phosphorylation of VEGF-2 so that VEGF cannot normally play the role of promoting angiogenesis after binding

2 Terminalia chebula
Retz.

$\mathrm{He} \mathrm{Zi}$ $x^{2}+x$ Junzi

Fruit to the receptor, thereby inhibiting the progression of DN. In addition, chebula extract can reduce the blood sugar of diabetic rats caused by STZ and improve the pathological tissue morphology of the kidney in diabetic rats [11].

3 Malva verticillata L. Dong Kui

4 Rubia cordifolia L. Qiancao घぶ`ラ| Rubiaceae Root

\begin{tabular}{|c|c|}
\hline 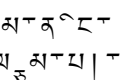 & Malvaceae \\
\hline
\end{tabular}
Malva seed is a commonly used drug
for the treatment of DN water stasis interaction syndrome [12].

R. cordifolia aqueous root extract exhibited significant

antihyperglycemic activities in STZinduced hyperglycemic rats [13]. Madder mixture can reduce the proteinuria of early DN and is effective in the treatment of early DN [14].

T. terrestris has antidiabetic and renal protective capability in alloxaninduced diabetic mice [15]. T. terrestris hydroalcoholic extract can reduce the total protein and albumin content of urine in diabetic rats induced by STZ, thereby improving diabetic kidney damage, and has the effect of treating DN [16].

Oral administration of $P$. longum dried fruits has shown significant antihyperglycemic, antilipid peroxidative, and antioxidant effects in diabetic rats [17]. The water extract of $P$. longum root has antidiabetic and antihyperlipidemic activities in the STZ-induced diabetes model in rats and also has a protective effect on diabetes-induced kidney damage [18]. 
TABLE 1: Continued.

$7 \quad$ Punica granatum L. Shiliu $\mathbb{N}^{\top} \vee \square_{エ \cup \mid}$ Punicaceae Fruit
Berberis kansuensis Schneid

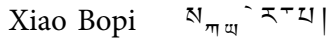

Berberidaceae

Endothelium
Pomegranate peel tannin can lower the blood sugar of diabetic rats, reduce the oxidation of free radicals on the structure of kidney tissue, and have a beneficial effect on the kidneys of diabetic rats [19]. Pomegranate peel tannin can alleviate the oxidative stress in the renal cortex of diabetic rats, protect the kidneys. and delay the occurrence of DN [20]. Methanolic extract of $P$. granatum leaves has potential antioxidant, antihyperglycemic, and antiglycosylation activities, helping slow down the progression of DN [21].

Berberine can improve the damage of vascular endothelial cells,

downregulate the expression of VEGF, reduce vascular permeability, protect

the function of microvascular endothelial cells, and improve the development of DN [22]. Berberis can upregulate the ornithine content in the serum of DN rats and participate in the metabolism of arginine and proline, thus improving the pathological changes and pharmacodynamic indicators of DN [23].

Mangiferin can remarkably ameliorate $\mathrm{DN}$ in rats by increasing the activity of glyoxalase 1 [24]. Mangiferin delayed the progression of DN and protected the podocytes by enhancing autophagy under diabetic conditions via the AMPK-mTOR-ULK1 pathway [25]. Mangiferin can reduce the degree of oxidative stress in the kidney and downregulate the expression of CTGF protein in the kidney, delaying kidney damage in diabetic rats [26].

Cinnamon and cinnamon-containing compounds can improve type 2 diabetes and its complications [27]. Cinnamon volatile oil can target to activate E2-related factor 2 (Nrf2), improve metabolic disorders caused by $\mathrm{DN}$, and protect kidney function [28]. The cinnamon extract can reduce diabetic kidney damage by controlling blood sugar, reducing oxidative stress and improving endothelial cell function [29]. 
TABle 1: Continued.

Rhizome

Polygonatum polysaccharide may inhibit the expression of ET-1 and

TGF-Pi in diabetic rat models, reduce ECM, delay the occurrence of renal fibrosis, and protect the kidneys of diabetic rats [30]. Polygonatum saponins can inhibit the process of renal tubulointerstitial fibrosis by blocking the activation of the Wnt/ $\beta$-catenin signaling pathway and ultimately play a role in protecting the kidneys of DN rats, which can be used for the prevention and treatment of DN [31, 32].

The triterpenoids oleanolic acid (OA) and maslinic acid (MA) in syzygium aromaticum can reduce postprandial hyperglycemia in diabetic rats induced by streptozotocin [33]. OA can enhance the renal function of diabetic rats induced by STZ [34].

In the treatment of DN, Angelica can reduce urine protein, regulate the expression level of related cytokines, reduce kidney damage, and improve renal function [35]. Angelica polysaccharides can inhibit the excessive proliferation of glomerular mesangium, reduce inflammation, and have a good effect on preventing and treating DN [36]. Angelica

polysaccharides can inhibit the differentiation of renal tubular epithelial cells, regulate the production and degradation of extracellular matrix components, and delay the development of diabetic renal fibrosis by reducing the activity of the TGF- $\beta 1$ / Smads signaling pathway [37]. Chinese herbal compound containing angelica has certain curative effects in improving renal function and inhibiting the development of DN [38].

Cassia can significantly inhibit the activation of NF-KB and the expression of fibronectin in rats, reduce glomerular hypertrophy, mesangial cell proliferation and extracellular matrix accumulation, and have a significant

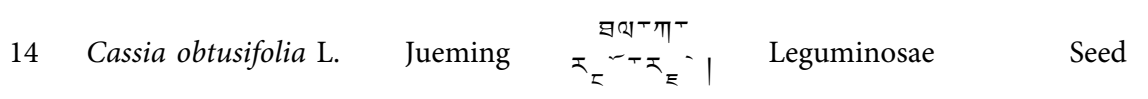
preventive and therapeutic effect on

DN [39]. Cassia anthraquinone glycosides can inhibit the expression of renin and AngII, reduce the content of KIM- 1 and $\beta 2-$ MG in urine, and have a protective effect on kidney injury in diabetic rats [40].

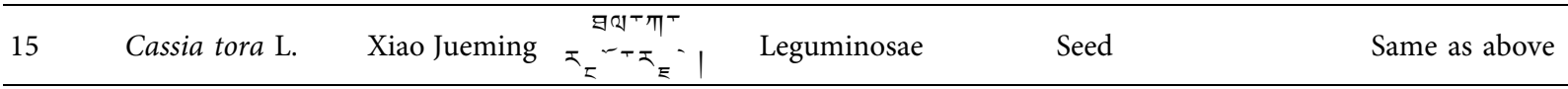


TABLE 1: Continued.

16

Lycium chinense Miller

$$
\text { ×こェー }
$$

Solanaceae

Fruit
Lycium barbarum polysaccharide (LBP) can reduce the production of glycosylation end products in model rats and reduce the secretion of renal IL-8, thus preventing the occurrence of DN [41]. LBP can reduce the expression of MCP1mRNA and ICAM-1mRNA by inhibiting the expression of NF- $\kappa$ B and AngII; it has a significant protective effect on the renal function of DN rabbits and delays the appearance and development of DN [42]. LBP can inhibit the damage of renal tubular epithelial cells in a high glucose environment, inhibit its inflammatory response, and improve the renal function damage of DN rats [43]. LBP can improve the blood sugar level, renal function, and pathological changes of kidney tissue in rats with diabetic kidney injury and has a protective effect on kidney tissue [44].

Lycium barbarum seed oil can significantly improve kidney function, control the kidney hypertrophy of diabetic mice, and can be used for the treatment of diabetic kidney injury [45].

Astragaloside may increase the autophagy activity of renal tissue cells by inhibiting PI3K/Akt/FoxO1 signal and slow down the development of type $2 \mathrm{DN}$ [46]. Astragaloside significantly inhibits renal endoplasmic reticulum stress, relieves CHOPmediated excessive apoptosis of renal tissue cells, significantly reduces proteinuria in $\mathrm{DN}$ rats, and improves renal tissue pathological damage in rats

[47]. Astragalus can prevent the progression of DN [48].

Trigonella tibetana can significantly reduce the levels of El, rVIIB2, and blood sugar in diabetic rats; effectively improve DN renal hemodynamics; enhance antioxidant capacity, thereby reducing blood sugar and urine microalbumin; and protect kidney function [49]. T. tibetana combined with valsartan in the treatment of patients with DN can significantly reduce their albumin level, which is more effective than valsartan alone, suggesting that $T$. tibetana can be an option for the treatment of DN [50]. 
TABle 1: Continued.

Picrorhiza scrophulariiflora

Pennell.
$\mathrm{Hu}$

Huanglian

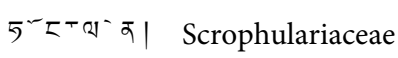

The total glycosides of rhizoma picrorhizae can significantly improve the hypertrophy of mesangial cells induced by high glucose, reduce the content of intracellular ROS, and increase the level of MMP and reduce $\mathrm{Ca}^{2+}$, so as to protect the oxidative stress damage of mesangial cells induced by high glucose [51]. The water extract of rhizoma picrorhizae has a certain curative effect on DN in rats $[52,53]$.

$R$. laevigata extract can improve glucose and lipid metabolism, renal dysfunction, and renal pathological

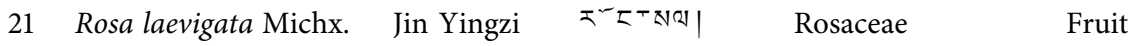
changes in DN rats; delay or prevent the development of $\mathrm{DN}$; and protect the renal function of diabetic rats $[54,55]$.

$P$. lingua flavonoids can reduce the level of inflammatory factors in the kidney tissue of diabetic rats and effectively improve the kidney injury and inflammatory response of $\mathrm{DN}$ [56].

Hematoxylon decoction can significantly reduce blood sugar, blood urea nitrogen and creatinine levels in DN rats, reduce kidney damage, and significantly improve kidney function [57]. Hematoxylon can significantly reduce blood CRPI and IL-6 levels in

$\mathrm{DN}$ rats, reduce urinary protein excretion, improve renal tissue morphology, and may protect the kidneys of early DN rats by inhibiting inflammation [58].

A. oxyphylla can improve the pathological state of the kidneys and

regulate metabolomics and the function of intestinal microbes, so as to achieve the purpose of treating DN

[59]. A. oxyphylla decoction can effectively lower blood sugar and reduce the excretion of urinary microalbumin, showing good renal protection, and it has a significant effect in the early treatment of DN. It can be used in the clinical treatment of DN $[60,61]$.

Plantain water extract can significantly reduce the degree of kidney damage in DN rats, and its mechanism may be related to the inhibition of the p38

Plantago depressa Willd.
Che Qiancao घะม।

Plantaginaceae
Whole grass MAPK pathway and activation of the PPAR- $\gamma$ pathway [62]. Plantain water extract can significantly reduce kidney damage in $\mathrm{DN}$ rats and reduce renal fibrosis [63]. 
TABle 1: Continued.

26 leontopodioides
(Willd.) Beauv.

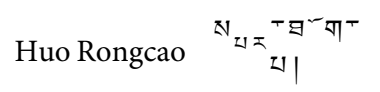

Compositae

Whole grass subset and the larval carcass

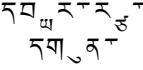

$$
\begin{aligned}
& \text { रू। }
\end{aligned}
$$

Ergotaceae
Edelweiss quercetin has a significant inhibitory effect on the lipid peroxidation in the kidney tissue of diabetic rats, significantly reduces urinary albumin excretion, improves the glomerular basement membrane and matrix membrane hyperplasia, and can be used as a potential therapeutic drug for DN [64].

Cordyceps can reduce urine protein and improve renal function in patients with DN [65]. Cordyceps can effectively alleviate renal tubular
Cordyceps sinensis (Berk.) Sacc. damage and renal tubular epithelial cell shedding and death, regulate the AMPK/mTOR signaling pathway related to autophagy in renal tubular epithelial cells, and have a protective effect on the kidneys of DN rats [66].

Soy isoflavones can protect the kidneys of diabetic rats by improving lipid metabolism disorders [67]. Soy isoflavones have anti-inflammatory and antioxidant effects, which can effectively protect the oxidative stress and inflammation in the kidneys of DN rats [68].

F. vulgare water extract can significantly improve the activity of kidney tissue antioxidant enzymes and the ability to scavenge oxygen free radicals, and reduce the oxidative stress damage of oxygen free radicals to the kidney tissue of diabetic rats, thereby protecting DN [69].

$B$. juncea significantly prevented the rise in creatinine levels; it will delay the development of DN [70].

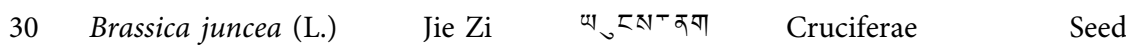

Sesame can improve kidney damage in diabetic rats and increase protein levels [71].

\begin{tabular}{|c|c|c|c|c|c|}
\hline Sesamum indicum L. & Hei Zhima & $5^{\circ}$ ग & Pedaliaceae & Seed & diabetic rats and incre \\
\hline
\end{tabular}

\begin{tabular}{|c|c|c|c|c|c|}
\hline & & & $\mathbf{D}^{\prime-} \bar{\alpha}^{\top} \mathrm{N}_{2} \bar{\alpha}^{\top}$ & & \\
\hline 32 & Dolichos lablab L. & Bian Dou & 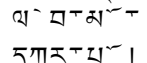 & Leguminosae & Seed \\
\hline
\end{tabular}

Dolichos lablab is a commonly used drug for the treatment of $\mathrm{DN}$ water stasis interaction syndrome [12].

$P$. radiatus significantly decreased glucose and increased insulin levels,

33 Phaseolus radiatus L. Cai Dou Leguminosae Seed
ameliorating the loss of renal function observed in STZ-induced diabetic rats [72].

34 \begin{tabular}{c}
$\begin{array}{c}\text { Rubia membranacea } \\
\text { (Franch.) Diels. }\end{array}$ \\
\hline
\end{tabular}

4.2.3. Tribulus terrestris. This is the mature fruit of the Tribulaceae plant $T$. terrestris L. (Tibetan name: गा $\exists^{-\top}$ (transliterated as Se-ma) and English name: Tribulus). Mostly born on barren hills and fields, distributed in sandy land in Tibet and other regions. Se-ma is sweet, astringent in taste and warm in nature and functions to benefit water and dispel dampness. It is mainly used for kidney heat, urinary atresia, malnourished edema, and other diseases. Rgyud bzhi records that se-ma can treat diuresis and cure rheumatoid arthritis and kidney disease.

Tribulus mainly contains flavonoids, saponins, amides, and other compounds [82]; the current pharmacological research mainly focuses on $T$. terrestris saponins. T. terrestris saponins have pharmacological effects of 


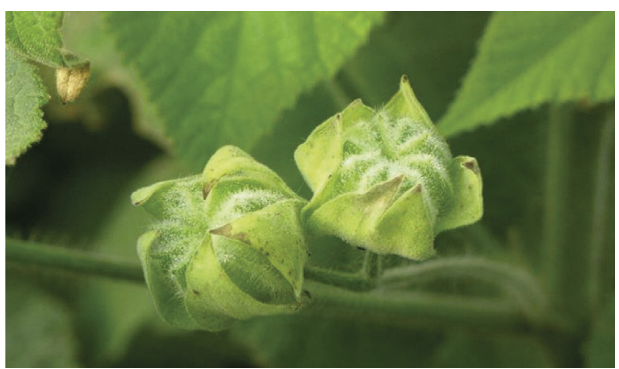

(a)

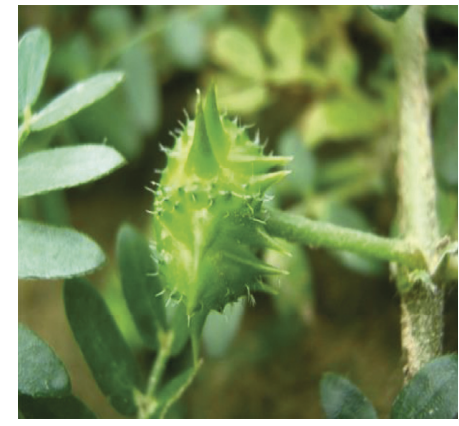

(c)

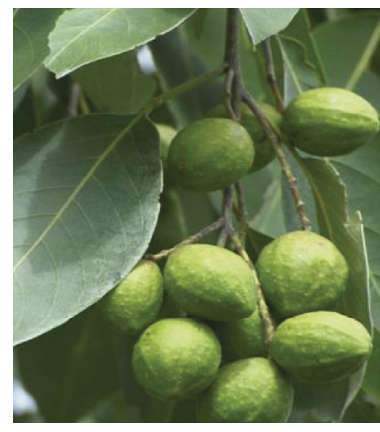

(e)

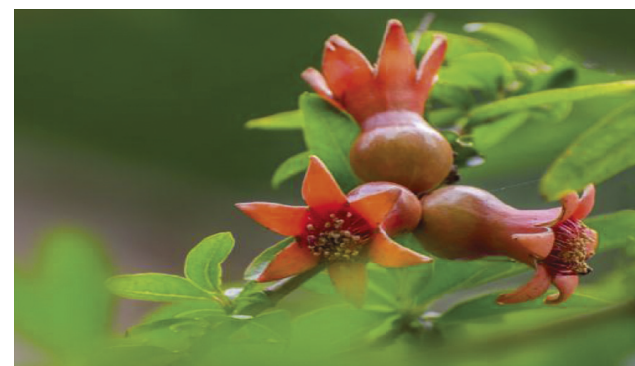

(b)

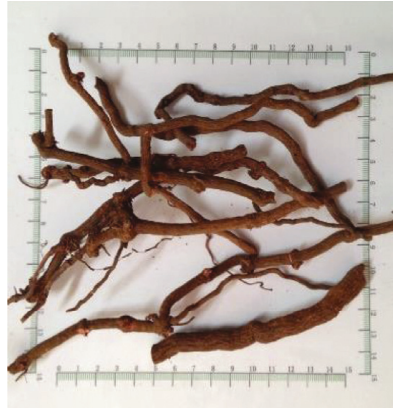

(d)

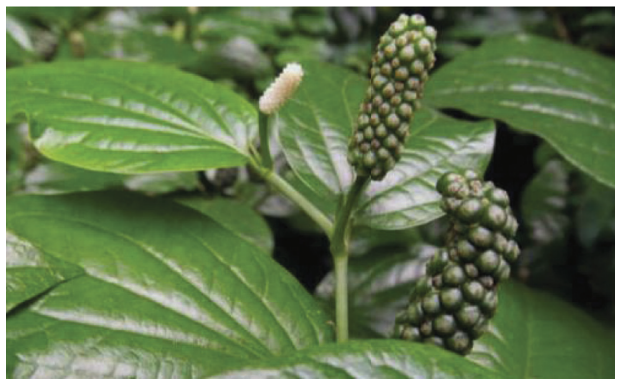

(f)

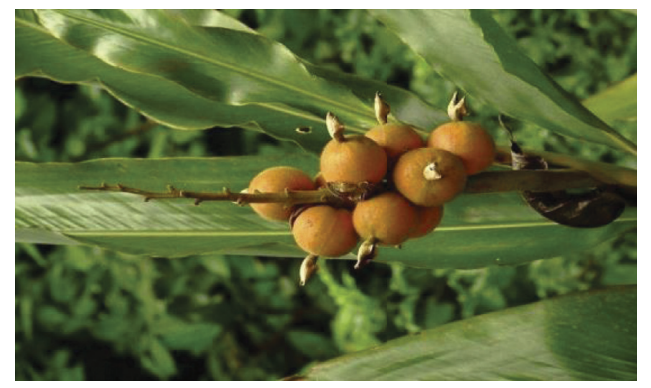

(g)

Figure 2: Commonly used Tibetan medicines for the treatment of DN: (a) Malva verticillata L., (b) Punica granatum L., (c) Tribulus terrestris L., (d) Rubia cordifolia L., (e) Terminalia chebula Retz., (f) Piper longum L., and (g) Amomum kravanh Pierre ex Gagnep.

antifatigue, protect myocardium, lower blood lipids, lower blood pressure, lower blood sugar, and so on [83]. Yang et al. [84] evaluated the effects of $T$. terrestris in preventing and treating renal calcium oxalate stones and found that blood urea nitrogen, creatinine, urine oxalic acid, $\mathrm{Ca}^{2+}$, and malondialdehyde contents of renal homogenate were increased, while blood $\mathrm{Ca}^{2+}$ and renal GPX content decreased significantly, renal calcium oxalate crystals was decreased, and kidney damage was alleviated after $T$. terrestris treatment. It is concluded that $T$. terrestris can effectively prevent and treat kidney calcium oxalate stones in rats and protect kidney function. Meng et al. [85] found that Tribulus can reduce the expression of LepR in the kidney, inhibit the response of the JAK2/STAT3 pathway, reduce the sensitivity of the kidney to leptin, reduce selective leptin resistance, lower blood pressure, and protect the kidney. Lamba 
et al. [86] found that the ethanol extract of T. terrestris had a significant protective effect on streptozotocin-induced diabetic rats by inhibiting oxidative stress. Zhao et al. [87] showed that $T$. terrestris extract can reduce TLR2 and TLR4 mRNA levels in pancreatic islet cells, inhibit inflammation, and significantly improve the clinical symptoms of polydipsia and polyphagia, which can be used for the treatment of type 2 diabetes. Zhao et al. [88] found that T. terrestris saponins also have a certain protective effect on the retina of type 2 diabetic rats. It has been reported that the hydroalcoholic extract of $T$. terrestris is able to reduce the urinary total protein and albumin content in streptozotocin-induced diabetic rats, thereby improving diabetic kidney damage and being used in the treatment of DN. In addition, among Professor Nian Li's 158 prescriptions for the treatment of DN, T. terrestris is the fifth most commonly used drug for the treatment of DN [89].

In summary, the high-frequency drugs used in the Tibetan medicine system for the treatment of kidney diseases are also commonly used drugs for the treatment of kidney diseases, diabetes, and DN in modern medicine. It should invest more on research to clarify its effects on DN and its mechanisms, so as to improve the safety and effectiveness of the clinical application.

\section{Deficiencies and Prospects}

Although Tibetan medicine has a positive effect on the treatment of DN, there are still many gaps and limitations in the research of Tibetan medicine. First of all, there are 362 kinds of Tibetan medicines in the Tibetan medicine system that can be used for the treatment of kidney diseases. Only 60 kinds have been proved to have kidney-related pharmacological activities; 34 kinds can be used for the treatment of DN; and most drugs still lack experimental evidence for the treatment of DN. For example, Malva seed is commonly used in the Tibetan medicine system to treat nephropathy, and it is also used by many physicians in the treatment of $\mathrm{DN}$. However, there is no research on its biologically active ingredients and pharmacological mechanisms in the treatment of DN. Therefore, it is necessary to carry out deep researches combined with modern medical research methods to clarify the bioactive components of these drugs and their action and mechanism on DN, so as to be used in the clinical treatment of DN as soon as possible. Secondly, we found that among the 60 kinds of Tibetan drugs for treating kidney diseases, 16 have antidiabetic activity, but there is no research report on DN. For example, modern research has found that Althaea rosea can be used for the treatment of kidney stones, kidney damage, and so on. The hypoglycemic and antioxidant capacity of its seeds indicate that it has a certain effect in the treatment of diabetes and its complications [90, 91], but there is no experimental research on $\mathrm{DN}$ at present. Therefore, we hope to invest some researches on these drugs to determine their effects on DN and find more drugs for the treatment of DN. In addition, the potential toxicity of Tibetan medicines should not be overlooked. For example, Radix Rubiae has a good therapeutic effect on $\mathrm{DN}$, but some studies have found that the alcohol extract of Radix Rubiae has slight hepatotoxicity and nephrotoxicity [92]. Thus, while using these drugs to treat diseases, the same attention should be paid to the evaluation of their potential toxicity, and certain processing or compatibility methods can be used to improve the safety of the clinical medication.

\section{Discussion}

Tibetan medicine is an important part of Chinese traditional medicine and excellent traditional culture, and it is one of the most influential ethnic medicine in China, which possesses a complete theoretical system and has rich experience in the treatment of "jingnisaku disease." By regulating the balance of the three major factors of rLung, Bad-kan, and mKhris-pa, Tibetan medicine put forward unique treatment methods against the "jingnisaku disease" from the aspects of drugs, diet, and daily life, which has considerable prospects for the treatment of diabetes mellitus and its complication DN. At the same time, we think that the occurrence of "jingnisaku disease" is closely related to the weakening of renal function through the understanding of Tibetan medicine. And modern medicine believes that $\mathrm{DN}$ is a secondary kidney disease caused by diabetes mellitus. For the treatment of $\mathrm{DN}$, consideration should be given to renal lesions while treating diabetes. Therefore, the occurrence and development of DN can be effectively prevented and treated by improving renal function and protecting the kidneys.

In view of the knowledge of Tibetan medicine on DN, the search for Tibetan medicines to treat $\mathrm{DN}$ will become an idea of new drug development. The results show that there are 60 kinds of drugs for the treatment of kidney diseases in the Tibetan medicine system, and 34 kinds of them have been used in the treatment of DN. These Tibetan medicines are all derived from botanicals and are mainly distributed in 23 families, among which the most frequently used is the leguminous family. It is worth mentioning that A. kravanh, T. chebula, M. verticillata, $R$. cordifolia, T. terrestris, P. longum, P. granatum, and other Tibetan medicines that are most commonly used to treat kidney diseases can also be used in the treatment of DN. Therefore, drugs for the treatment of kidney disease are expected to become a potential source of drugs for the treatment of DN. We find that these drugs mainly exert their effects in the treatment of DN by improving kidney function, lowering blood sugar and blood lipids, and reducing urine protein content, anti-inflammatory, and antioxidative stress and other pathways. However, the mechanisms of some drugs are not yet clear; multidisciplinary approaches should be integrated to perform more pharmacological studies to reveal their mechanisms of action.

To sum up, this study finds that drugs for the treatment of kidney disease can be used as potential drug candidates for the treatment of DN and sorts out the drugs used by Tibetan medicine in the treatment of DN, which expanded the scope of DN medication and provided a new idea for the treatment of DN. In order to make better use of Tibetan medicines, it is necessary to conduct in-depth research of 
existing Tibetan medicines combined with modern pharmacology, phytochemistry, and other methods to clarify the bioactive ingredients and mechanism of potential drugs for $\mathrm{DN}$ and to evaluate their toxic and side effects to improve the effectiveness and safety of Tibetan medicines in the treatment of DN.

\section{Conflicts of Interest}

The authors declare that there are no conflicts of interest.

\section{Authors' Contributions}

Lili $\mathrm{Pu}$ conducted the research; performed data analysis; collected, organized, and wrote the Tibetan names of natural medicines; and wrote the paper. Xianrong Lai conceived and designed the study. Chunhong Yang, Liqiong Yu, Shiling Li, Yaqin Liu, and Xinan Liu amended the paper.

\section{Acknowledgments}

This research was funded by the National Natural Science Foundation of China (no. 81473427) and National Key R\&D Project (2017YFC1703900).

\section{References}

[1] L. Zhang, J. Long, W. Jiang et al., "Trends in chronic kidney disease in China," New England Journal of Medicine, vol. 375, no. 9, pp. 905-906, 2016.

[2] A. T. Reutens and R. C. Atkins, "Epidemiology of diabetic nephropathy," Contributions to Nephrology, vol. 170, pp. 1-7, 2011.

[3] Z. D. Gong and C. J. Li, "Tradition Chinese medicine understanding of the etiology, pathogenesis and treatment of diabetic renal edema," Chinese Medicine Journal, vol. 31, no. 04, pp. 494-498, 2016.

[4] K. Umanath and J. B. Lewis, "Update on diabetic nephropathy: core curriculum 2018," American Journal of Kidney Diseases, vol. 71, no. 6, pp. 884-895, 2018.

[5] K. Daiji, M. Keiichiro, T. Yusuke et al., "SGLT2 inhibitors as a therapeutic option for diabetic nephropathy," International Journal of Molecular Sciences, vol. 18, no. 5, p. 1083, 2017.

[6] American Diabetes Association(ADA), "Standard of medical care in diabetes-2017," Diabetes Care, vol. 40, no. suppl 1, pp. s4-s128, 2017.

[7] C. Y. Wang and Z. S. Jin, "To explore the therapeutic mechanism of diabetic nephropathy based on vitamin D axis," Journal of Practical Traditional Chinese Medicine, http://lib. cdutcm.edu.cn:29006/kcms/detail, 2021.

[8] D. Wang, Y. X. Li, and J. L. Li, "Treating diabetic nephropathy from deficiency of spleen and kidney," Liaoning Journal of Traditional Chinese Medicine, vol. 44, no. 1, pp. 60-62, 2017.

[9] J. Gao, L. Pan, R. Bi et al., "Tibetan medicines and Tibetan prescriptions for the treatment of diabetes mellitus," Evidence-based Complementary and Alternative Medicine, vol. 2021, Article ID 5532159, 2021.

[10] H. M. Chen, D. N. B. Q. Su, and C. Chang, "Protective effect of white cardamom volatile oil on kidney in diabetic nephropathy rats," Chinese Journal of Traditional Chinese Medicine, vol. 32, no. 9, pp. 4227-4230, 2017.
[11] B. Li, X. Li, and Y. Fan, "Research progress on pharmacological effects of myrobalan," Pharmaceutical Research, vol. 34, no. 10, pp. 591-595, 2015.

[12] S. T. Lu, Q. G. Chen, and P. Y. Xu, "Discussion on Ding Xueping's famous TCM medication rule for diagnosis and treatment of diabetic nephropathy based on the auxiliary platform of traditional Chinese medicine inheritance," New Chinese Medicine, vol. 49, no. 4, pp. 203-207, 2017.

[13] R. Baskar, L. M. Bhakshu, G. Vijaya Bharathi et al., "Antihyperglycemic activity of aqueous root extract of Rubia cordifolia. In streptozotocin-induced diabetic rats," Pharmaceutical Biology, vol. 44, no. 6, pp. 475-479, 2006.

[14] B. Ying and Q. Du, "Treating 30 cases of early diabetic nephropathy with qiancao mixture," Journal of Practical Traditional Chinese Medicine, vol. 19, no. 6, p. 543, 2005.

[15] A. Istiak, P. Hazra, S. Das, M. I. Hossain, A. S. Aminatu, and K. Rafiq, "Hypoglycemic, hypolipidemic and kidney protective potential of combined formulation of Tribulus terrestris and Andrographis paniculata in alloxan induced mice," African Journal of Pharmacy and Pharmacology, vol. 12, no. 21, pp. 269-277, 2018.

[16] D. Tong, Study on the Mechanism and Compatibility of Tibetan Medicine Siwei Jianghuang Decoction on Diabetic Nephropathy, Chengdu University of Traditional Chinese Medicine, Chengdu, China, 2018.

[17] S. Manoharan, S. Silvan, K. Vasudevan, and S. Balakrishnan, "Antihyperglycemic and antilipidperoxidative effects of Piper longum, dried fruits in alloxan induced diabetic rat," The Journal of Biological Sciences, vol. 7, no. 1, pp. 161-168, 2007.

[18] A. N. Shaik, B. K. R amesh, S. Swapna, K. T. Thandaiah, V. J. K. Malaka, and A. R. Chippada, "Antidiabetic and antihyperlipidemic activity of Piper longum root aqueous extract in STZ induced diabetic rats," BMC Complementary and Alternative Medicine, vol. 13, no. 37, pp. 37-46, 2013.

[19] H. J. Tan, L. Yang, and M. Z. Wang, "Study on the antioxidant protective effect of pomegranate peel tannin on kidney tissue of diabetic rats," Journal of Toxicology, vol. 27, no. 5, pp. 356-359, 2013.

[20] L. Yang, "Study on the Protective Effect of Pomegranate Peel Tannin on the Kidneys of Diabetic Nephropathy Rats," Hubei Three Gorges Vocational and Technical College, Yichang, China, 2010.

[21] S. N. Mestry, J. B. Dhodi, S. B. Kumbhar, and A. R. Juvekar, "Attenuation of diabetic nephropathy in streptozotocin-induced diabetic rats by Punica granatum Linn. leaves extract," Journal of Traditional and Complementary Medicine, vol. 7, no. 3, pp. 273-280, 2017.

[22] Y. Zhao, H. Feng, Z. Zhou et al., "Ideas for new drug discovery of prevention and treatment of diabetic nephropathy based on "holistic view" in Tibetan medicine," Chinese Journal of Experimental Formulas, vol. 25, no. 3, pp. 167-172, 2019.

[23] X. P. Ai, X. B. Wang, and X. Y. Wang, "Evaluation on the protection mechanism of Berberidis dictyophyllae cortex on STZ-induced diabetic nephropathy in rats based on metabolomics," Pharmacology and Clinics of Chinese Medicine, vol. 35, no. 2, pp. 67-73, 2019.

[24] Y. W. Liu, X. Zhu, L. Zhang et al., "Up-regulation of glyoxalase I by mangiferin prevents diabetic nephropathy progression in streptozotocin-induced diabetic rats," European Journal of Pharmacology, vol. 721, pp. 355-364, 2013.

[25] X. Wang, L. Gao, H. Lin et al., "Mangiferin prevents diabetic nephropathy progression and protects podocyte function via autophagy in diabetic rat glomeruli," European Journal of Pharmacology, vol. 824, no. 5, pp. 170-178, 2018. 
[26] C. Zhang, J. S. Wang, G. B. Zhang, and S. Li, "Protective effects of mangiferin on the diabetic nephropathy in streptozotocininduced diabetic rats," Journal of Wannan Medical College, vol. 36, no. 4, pp. 314-317, 2017.

[27] R. F. Ma, L. L. Wang, and Y. B. Guo, "Theoretical basis and clinical research of the treatment of type 2 diabetes mellitus with cinnamon," Chinese Journal of Basic Chinese Medicine, vol. 22, no. 3, pp. 409-411, 2016.

[28] H. Zheng, S. A. Whitman, W. Wu et al., "Therapeutic potential of Nrf2 activators in streptozotocin-induced diabetic nephropathy," Diabetes, vol. 60, no. 11, pp. 3055-3066, 2011.

[29] F. Wu, C. Gong, and Z. Y. Chen, "Protective effect of methylhydroxy chalcone polymer extracted from Cassia on the renal damage inunilaterally nephrectomized diabetic rats," Chinese Journal of Experimental Surgery, vol. 45, no. 11, pp. 1515-1518+1584, 2009.

[30] T. T. Fu, Protective Effect of Polygonatum Polysaccharide on Kidney Injury in Diabetic Rats, Jinzhou Medical University, Jinzhou, China, 2016.

[31] J. Peng, "Protective effect of Polygonatum saponins on kidney injury in diabetic nephropathy rats and the effect of Wnt/ $\beta$-catenin signaling pathway," Chinese Traditional Patent Medicine, vol. 41, no. 10, pp. 2518-2521, 2019.

[32] Z. G. Zhou, M. H. Gong, and D. H. Li, "Research progress on pharmacological action and mechanism of polygonatum sibiricum polysaccharide," Journal of Liaoning University of Traditional Chinese Medicine, http://kns.cnki.net/kcms/ detail/21.1543.R.2021041\%206.1828.059.html, 2021.

[33] K. Andile, M. R. Serumula, R. B. Myburg, F. R. Van Heerden, and C. T. Musabayane, "Effects of Syzygium aromaticumderived triterpenes on postprandial blood glucose in streptozotocin-induced diabetic rats following carbohydrate challenge," PloS One, vol. 8, no. 11, Article ID e81632, 2013.

[34] P. M. Hlengiwe, B. Masola, M. Singh, and C. T. Musabayane, "The effects of Syzygium aromaticum-derived oleanolic acid on kidney function of male sprague-dawley rats and on kidney and liver cell lines," Renal Failure, vol. 34, pp. 767-776, 2012.

[35] X. D. Ren and Y. W. Zhang, "Research progress of huangqi (Astragalus), danggui (angelica) and their compounds in treatment of diabetic nephropathy," Chinese Journal of Traditional Chinese Medicine, vol. 33, no. 10, pp. 2318-2320, 2015.

[36] Y. Sui, W. Liu, W. Tian, and X.-Q. Li, W. Cao, “A branched arabinoglucan from Angelicae Sinensis Radix ameliorates diabetic renal damage in rats," Phytotherapy Research, vol. 33, no. 3, pp. 818-831, 2019.

[37] Y. Q. Shi, Based On the TGF- $\beta 1 /$ Smad Signaling Pathway to Explore the Mechanism of Angelica Polysaccharide in Intervening Diabetic Nephropathy, Lanzhou University, Lanzhou, China, 2018.

[38] Z. M. Chen, R. K. Li, and S. F. Wan, "Clinical research of Chinese angelica compound in the treatment of diabetic nephropathy," Asia-Pacific Traditional Medicine, vol. 11, no. 24, pp. 72-74, 2015.

[39] L. Li, M. Z. Yang, and Y. Q. Chen, "Efficacy observation of Cassia seed on diabetic nephropathy of experimental rats," Chinese Journal of Integrated Traditional Chinese and Western Medicine, vol. S1, no. 25, pp. 71-72, 2006.

[40] Y. M. Song, "The protective effect of cassia glycoside on renal injury in diabetic rat rats," Clinical Journal of Chinese Medicine, vol. 10, no. 20, pp. 6-7, 2018.

[41] G. J. Sun, Y. Zhang, and J. Huang, "Study on the protective effect of lycium barbarum polysaccharides on kidney and its mechanism in type II diabetic rats," Acta Nutrimenta Sinica, vol. 1, no. 01, pp. 47-50, 2006.

[42] Q. Zhao, J. Li, J. Yan et al., "Lycium barbarum polysaccharides ameliorates renal injury and inflammatory reaction in alloxan-induced diabetic nephropathy rabbits," Life Sciences, vol. 157, pp. 82-90, 2016.

[43] F. X. Qu, Y. Y. Bian, and Y. Yu, "Lycium barbarum polysaccharide inhibits inflammatory response in rats with diabetic nephropathy via TLR4/myd88 pathway," Progress of Anatomical Sciences, vol. 26, no. 04, pp. 378-382, 2020.

[44] N. Zhang, Protective Effect and Mechanism of Lycium Barbarum Polysaccharides on Diabetic Renal Injury, Jinzhou Medical University, Jinzhou, China, 2020.

[45] W. B. Wang, H. B. Li, and J. Wu, "Interventional effect of Lycium barbarum seed oil on kidney injury in C57BL/6J mice with type 2 diabetes," Journal of Ningxia Medical University, vol. 34, no. 1, pp. 1-3, 2012.

[46] K. K. Ma, Y. H. Ju, Q. Q. Chen, W.-zu LI, and W.-ping LI, "Effect of astragaloside IV on regulation of PI3K/Akt/FoxO1 signal in kidney of type 2 diabetic nephropathy rats," Chinese Journal of Experimental Formulas, vol. 25, no. 2, pp. 74-81, 2019.

[47] H. Liu, Z. S. Wang, and W. Gao, "Influence of astragaloside IV on endoplasmic reticulum stress and CHOP signaling pathways in diabetic nephropathy rats induced with STZ," Chinese Journal of Hospital Pharmacy, http://kns.cnki.net/kcms/ detail/42.1204.R.20210510.1616.002.html, 2021.

[48] H. M. Ni, Z. Y. Dong, and X. M. Chen, "Research progress of the curative effects of main active components of Astragalus membranaceus in treatment of diabetic nephropathy," Medical Journal of Chinese People's Liberation Army, vol. 46, no. 03, pp. 294-299, 2021.

[49] Y. Ruan and C. F. Huang, "Experimental study on the protective effect of fenugreek on the kidney of diabetic rats," China Traditional Chinese Medicine Technology, vol. 15, no. 06, pp. 432-433, 2008.

[50] C. K. Guo and Q. Zhang, "Trigonella foenum graecum combined with valsartan in treatment of diabetic nephropathy," Chinese Journal of Difficult and Complicated Cases, vol. 11, no. 03, pp. 191-193, 2012.

[51] M. Sun, H. W. Fan, H. Y. Ma, and Q. Zhu, "Protective effect of total glucosides of picrorhiza scrophulariiflora against oxidative stress in glomerular mesangial cells induced by high glucose," Acta Pharmaceutica Sinica, vol. 42, no. 04, pp. 381-385, 2007.

[52] V. Patil, A. Bandivadekar, and D. Debjani, "Inhibition of Propionibacterium acnes lipase by extracts of Indian medicinal plants," International Journal of Cosmetic Science, vol. 34, no. 3, pp. 234-239, 2012.

[53] S. Zahirnddin, W. Khan, R. Nehra et al., "Pharmacokinetics and comparative metabolic profiling of iridoid enriched fraction of Picrorhiza kurroa-An Ayurvedic Herb," Journal of Ethnopharmacology, vol. 197, no. 2, pp. 157-164, 2016.

[54] C. Deng, H. W. Zhang, and Y. Jiang, "Inhibitory effects of Schisandra chinensis, Rosa laevigata and Cornus officinalis on glycometabolism in type 2 diabetes model rats," Natural product research and development, vol. 30, no. 4, pp. 568-574, 2018.

[55] Y. J. Zhou, Q. J. Liao, and T. P. Luo, "Renal protective effect of extracts from Rosa laevigata Mickx. on diabetic nephropathy rat," Progress in Modern Biomedicine, vol. 14, no. 36, pp. 7019-7024, 2014.

[56] X. L. Liu, W. P. Liu, L. L. Wang, and L. Feng, "Effects of flavonoids from Pyrrosiae folium on pathological changes and 
inflammatory response of diabetic nephropathy," China Journal of Chinese Materia Medica, vol. 43, no. 11, pp. 2352-2357, 2018.

[57] H. Zhnag, Z. Zhang, and X. B. Huang, "Effects of sappan decoction on blood sugar, renal function and renal pathology in rats with diabetic nephropathy," Lishizhen Medicine and Materia Medica Research, vol. 22, no. 05, pp. 1255-1256, 2011.

[58] J. K. Hu and H. W. Li, "Experimental Study on Hematoxylon in preventing and treating early phase diabetic nephropathy," Information on Traditional Chinese Medicine, vol. 28, no. 02, pp. 101-104, 2011.

[59] Y. L. Ni, Based on Intestinal Flora and Metabonomics Analysis of the Mechanism of Action of Yizhiren in the Treatment of Diabetic Nephropathy, Hainan Medical College, Haikou, China, 2019.

[60] Y. H. Song, K. Y. Yang, and X. W. Yue, "The effect of yizhiren decoction on diabetic nephropathy mice," Chinese Medicine Modern Distance Education of China, vol. 18, no. 07, pp. 100-102, 2020.

[61] K. Li, T. P. Ma, and K. Niu, "A review of the related studies of the prevention and treatment of diabetic nephropathy by the Alpinia oxyphylla," Chinese Medicine Modern Distance Education of China, vol. 19, no. 03, pp. 192-195, 2021.

[62] X. Q. Li, C. Wang, and H. Yang, "Study on the effect of plantain water extract on fibrosis in rats with diabetic nephropathy based on p38 MAPK/PPAR- $\gamma$ pathway," Chinese Journal of Integrated Traditional Chinese and Western Medicine Nephropathy, vol. 19, no. 10, pp. 885-887+945, 2018.

[63] K. Zhang and L. Zhao, "Effects of aqueous extract of plantain on renal fibrosis and p38 MAPK/peroxyppar- $\gamma$ pathway in diabetic nephropathy rats," Drugs and Clinic, vol. 17, no. 10, pp. 16-19, 2020.

[64] Y. J. Liu, H. Jiang, and Y. G. Jiang, "Leontopodium research survey," Journal of Liaoning University of TCM, vol. 12, no. 08, pp. 46-48, 2010.

[65] R. C. Chen, J. Q. Xiang, and H. Y. Chen, “A systematic review of the curative effect of cordyceps sinensis in treating diabetic nephropathy," Chinese Journal of Integrated Traditional Chinese and Western Medicine Nephropathy, vol. 18, no. 04, pp. 340-344, 2017.

[66] Z. Xu, K. Zhao, and Z. J. Li, "Cordyceps sinensis inhibits autophagy through AMPK/mTOR signaling pathway in rat renal tubular cell model," China Journal of Modern Medicine, vol. 28, no. 03, pp. 1-5, 2018.

[67] R. F. Li, C. Y. Cao, and Z. H. Liu, "Protective effect of soybean isoflavone on the kidney of diabetic rats and its mechanism," Central South Pharmacy, vol. 12, no. 06, pp. 532-534, 2014.

[68] X. Zhou, B. Li, and Z. H. Li, "Soy isoflavones protect kidney inflammation and oxidative stress injury in rats with diabetic nephropathy," Anatomy Research, vol. 41, no. 02, pp. 107-109+118, 2019.

[69] S. T. Li, C. J. Huang, and J. M. Wei, "Protective effect of Foeniculum vulgare extracts on kidney damage of diabetic rats," Guangxi Medical Journal, vol. 39, no. 09, pp. 1374-1377, 2017.

[70] J. K. Grover, S. P. Yadav, and V. Vats, "Effect of feeding Murraya koenigii and Brassica juncea diet kidney functions and glucose levels in streptozotocin diabetic mice," Journal of Ethnopharmacology, vol. 85, no. 1, pp. 1-5, 2003.

[71] P. Bhuvaneswari and S. Krishnakumari, "Nephroprotective effects of ethanolic extract of Sesamum indicum seeds (Linn.) in streptozotocin induced diabetic male albino rats," International Journal of Green Pharmacy, vol. 6, no. 4, 2012.
[72] C. Lomas-Soria, I. F. Pérez-Ramírez, J. Caballero-Pérez et al., "Cooked common beans (Phaseolus vulgaris L.) modulate renal genes in streptozotocin-induced diabetic rats," The Journal of Nutritional Biochemistry, vol. 26, no. 7, pp. 761-768, 2015.

[73] Y. Zhou, W. Li, and X. N. Qu, "Review on the distribution and biological activity of dihydrochalcone," Chinese Wild Plant Resources, vol. 33, no. 6, pp. 36-40, 2014.

[74] H. F. Yan, X. D. Dai, K. T. Fan, and Yi Wang, "Research on medication regularity of traditional Chinese medicine based on hyperuricemia patents," Chinese Herbal Medicines, vol. 8, pp. 376-381, 2016.

[75] X. L. Chen, S. S. Feng, and K. Zhang, "Effect of Amomurn kravanh volatile oil on the expression of caspase-3, Bax, Bcl-2 and NF-KB p65 in kidney tissues of rats with gentamicininduced acute kidney injury," Journal of Shihezi University, vol. 38, no. 05, pp. 629-634, 2020.

[76] H. M. Chen, X. L. Wang, and S. R. Enhe, "Effect of Amomum kravanh Pierre ex cagnep to expression of TGF-B1. PAI-1 on adriamycin induced renal injury rats," Pharmacology and Clinics of Chinese Medicine, vol. 33, no. 3, pp. 105-108, 2017.

[77] G. P. S. Kumar, P. Arulselvan, D. S. Kumar, and S. P. Subramanian, "Anti-diabetic activity of fruits of Terminalia chebula on streptozotocin induced diabetic rats," Journal of Health Science, vol. 52, no. 3, pp. 283-291, 2006.

[78] S. Y. Wu, X. W. Zhang, and L. Y. Yao, "Study on antioxidant activity of myrobalan," Jiangsu Agricultural Sciences, vol. 1, pp. 368-370, 2011.

[79] S. Tayal, S. Duggal, P. Bandyopadhyay, A. Aggarwal, S. Tandon, and C. Tandon, "Cytoprotective role of the aqueous extract of Terminalia chebula on renal epithelial cells," International Brazilian Journal of Urology, vol. 38, no. 2, pp. 204-213, 2012.

[80] N. K. Rao and S. Nammi, "Antidiabetic and renoprotective effects of the chloroform extract of Terminalia chebula Retz. seeds in streptozotocin-induced diabetic rats," BMC Complementary and Alternative Medicine, vol. 6, no. 1, pp. 1-6, 2006.

[81] J. H. Kim, C. O. Hong, Y. C. Koo, Su-J. Kim, and K.-W. Lee, "Oral Administration of ethyl acetate-soluble portion of Terminalia chebula conferring protection from streptozotocin-induced diabetic mellitus and its complications," Biological and Pharmaceutical Bulletin, vol. 34, no. 11, pp. 1702-1709, 2011.

[82] R. H. Li, Studies on Bioactive Compounds and Quality Evaluation of Tribulus terrestris L, Liaoning University of Traditional Chinese Medicine, Shenyang, China, 2006.

[83] S. Chhatre, T. Nesari, G. Somani, D. Kanchan, and S. Sathaye, "Phytopharmacological overview of Tribulus terrestris," Pharmacognosy Reviews, vol. 8, no. 15, p. 45, 2014.

[84] C. J. Yang, Y. J. Qin, and Y. W. Shan, "The prevention and treatment effect and mechanism of Tribulus terrestris on calcium oxalate calculus in rat kidney," International Journal of Surgery, vol. 46, no. 1, pp. 39-43, 2019.

[85] X. Q. Meng, Y. H. Jiang, S. Wu, and C.-H. Yang, "Pharmacodynamic and pharmacological effect of tribulus Ter5restris on kidney of rats with obesity-related hypertension through leptin mediated JAK2/STAT3 pathway," Chinese Traditional and Herbal Drugs, vol. 48, no. 03, pp. 539-545, 2017.

[86] H. S. Lamba, C. H. Bhargava, M. Thakur, and S. Bhargava, “ $\alpha$ glucosidase and aldose reductase inhibitory activity in vitro and antidiabetic activity in vivo of Tribulus terrestris," International Journal of Pharmacy and Pharmaceutical Sciences, vol. 3, pp. 270-272, 2011. 
[87] B. S. Zhao, P. S. Li, and S. Y. Zhang, "The efficacy of fructus tribuli extract JL201 on type 2 diabetes and its potential mechanisms," Chinese Clinical Pharmacology and Therapeutics, vol. 19, no. 9, pp. 1006-1010, 2014.

[88] Y. X. Wang, F. Wang, and S. Wang, "Protective effect of Tribulus terrestris saponins on retina and TrkB signaling pathway in type 2 diabetic rats," Medical Journal of Chinese People's Liberation Army, vol. 10, pp. 24-28, 2020.

[89] L. Nian, "Mining Professor NIAN Li's medication rules for diabetic nephropathy based on the software of "Traditional Chinese Medicine inheritance support system"," Tianjin Traditional Chinese Medicine, vol. 37, no. 10, pp. 1190-1194, 2020.

[90] N. Fahamiya, M. Shiffa, and M. Aslam, "A comprehensive review on Althaea rosea linn," Journal of Pharmaceutical Research, vol. 6, no. 11, 2016.

[91] P. A. Dar, F. Ali, I. A. Sheikh, S. A Ganie, and T. A. Dar, "Amelioration of hyperglycaemia and modulation of antioxidant status by Alcea rosea seeds in alloxan-induced diabetic rats," Pharmaceutical Biology, vol. 55, no. 1, pp. 1849-1855, 2017.

[92] Z. L. Zheng, S. Q. Li, and Y. P. Zhong, "Hepatotoxicity, Nephrotoxicity and Colonic Toxicity in Rats Induced by Rubiae Radix et Rhizoma Ethanol Extract," Chinese Journal of Experimental Formulas, vol. 23, no. 12, pp. 151-156, 2017. 